ISSN: 2641-9475

\title{
A Clinical Study of Biomarkers to Predict the Efficacy of Cancer Immunotherapy with Subcutaneous Low-Dose II-2 or with Im- mune Checkpoint Inhibitors
}

\author{
Lissoni P, Brivio F, Messina G, Vigore L, Rovelli F, Di Fede G \\ Institute of Biological Medicine, Milan, Italy
}

\section{"Corresponding author:}

\author{
Paolo Lissoni \\ Institute of Biological Medicine, \\ Milan, Italy
}

Received : February 22, 2019

Published : March 14, 2019

\begin{abstract}
The recent advances in the knowledge of the mechanisms involved in the antitumor immunity have allowed to elaborate immunotherapeutic strategies of cancer not only on the basis of an empiristic approach, as well as in the past years but on a better definition of the physiopathology of the anticancer immune response. In particular, it has been demonstrated that the antitumor immunity is mainly mediated by lymphocytes and that their functional status depends on the expression of specific cell-surface molecules, the so-called immune checkpoints, whose expression may block $T$ cell activation, the most important of them would be represented by PD-1 and its ligands, PD-L1 and PD-L2. Lymphocyte functions may be activated by IL-2, whereas the action of checkpoints may be blocked by specific anti-PD-1 monoclonal antibodies (MABs). Even though there are controversial results, the efficacy of anti-PD-1 MABs would be related to the expression of PD-L1 by tumor cells or at tumor microenvironment sites, whereas at present no patient-related biological response has been identified to predict the efficacy of anti-PD-1 MABs, whereas that of IL-2 immunotherapy may be predicted by the evidence of an important increase in lymphocyte count in association with a low macrophage system activation. Finally, recent observations have demonstrated that the interactions between lymphocyte and macrophage systems may be reflected by the simple lymphocyte-to-monocyte ratio (LMR) and that the evidence of an abnormally low LMR predicts a poor prognosis. The present study was performed to evaluate the relation between the efficacy of the anti-PD-1 MAB Nivolumab and patient biological response, as investigated by detecting lymphocyte, monocyte, eosinophil counts and LMR, in metastatic non-small cell lung cancer (NSCLC), by comparing the results to those obtained by SC low-dose IL-2. The study included 30 patients treated with Nivolumab and 20 patients treated with IL-2. Patients were evaluated before and after 3 months of immunotherapy. Nivolumab was intravenously administered at $3 \mathrm{mg} / \mathrm{kg}$ b.w. At 15-day intervals. IL-2 was injected subcutaneously at a dose of 6 MIU/day for 5 days/week for 4 consecutive
\end{abstract}


weeks, followed by 2 week-rest period. No significant differences in the percentages of both tumor regression and stable disease were seen between patients treated by Nivolumab or IL-2. Irrespectively of the clinical response, mean values of both lymphocytes and eosinophils significantly increased on IL-2 therapy. Monocyte mean count decreased on IL-2 therapy, without, however, significant differences. On the contrary, an increase in lymphocyte and eosinophil mean counts and a decline in monocyte number occurred in the only patients with tumor regression or stable disease under Nivolumab therapy, even though the difference was not significant. In contrast,

\section{INTRODUCTION}

Despite its complexity, today there is no doubt about the existence of a physiological immune-mediated antitumor biological resistance, whose mechanisms are finally almost completely defined. In fact, it has been demonstrated that the antitumor immunity is substantially a lymphocyte-dependent phenomenon [1]. All lymphocyte subsets play an anticancer role, the cytotoxic $\mathrm{T}$ lymphocytes by exerting an antigendependent cytotoxicity, the T helper-1 (TH1) lymphocytes by inducing a systemic activation of the immune system through the release of IL2 as a main T cell growth factor, and the NK cells, which may exert an antigen-independent cytotoxicity against fresh human cancer cells after their activation by IL-2 [2], with the only exception represented by the regulatory $T$ lymphocytes (T reg) [3], which in contrast may suppress the antitumor immunity namely through the production of TGFbeta, one of the most endogenous immunosuppressive factors [4]. Moreover, it has been demonstrated that the generation of $\mathrm{T}$ reg lymphocytes is stimulated by the macrophage-mediated chronic inflammatory response [5]. The functional status of the monocyte-macrophage system may be clinically investigated by monitoring the blood levels of several biomarkers of the chronic inflammatory response, the most important of them would be represented by CRP, neopterin, and soluble IL-2 receptor [6]. Moreover, it has recently been demonstrated that the simple monocyte count is positively correlated with the degree of macrophage system activation and with macrophage infiltration within the tumor mass, which has appeared to stimulate tumor cell proliferation and to predict a poor prognosis [5,7]. Then, being the anticancer immunity the end- result of the balance between two opposite biological dynamics consisting of the activation of an effective anticancer immune response, namely mediated by lymphocytes $[1,2]$, or its suppression induced by the macrophage-T reg cell system [3-5], the interaction between a significant increase in LMR mean values were observed in patients of Nivolumab group, who achieved a disease control on therapy. This preliminary study would suggest that the increase in LMR may represent a biomarker able to predict the efficacy of anti-PD-1 immunotherapies, while lymphocytosis constitutes the most important favorable prognostic biomarker on IL-2immunotherapy.

KEYWORDS: Checkpoint inhibitors, Immune checkpoints, Immunotherapy, interleukin-2, Nivolumab, Programmed Death-1 (PD-1), PD-L1.

activation and suppression of the anticancer immunity may be simply reflected and synthetized by the lymphocyte-tomonocyte ratio (LMR), whose normal values have appeared to be at least greater than 2.1 [8]. The prognostic importance of LMR is confirmed by the fact that the evidence of an abnormally low LMR value has appeared to predict a poor prognosis [8]. This finding is not surprising since lymphocyte and monocyte counts reflect the activation or the suppression, respectively, of the anticancer immunobiological response [1-4]. Moreover, it has to be remarked that lymphocyte-mediated antitumor activity depends on two fundamental variables, consisting of their absolute number and their functional status. From this point of view, it has recently been demonstrated that lymphocyte functional activation would be regulated by the expression of some particular cell surface molecules, the socalled immune checkpoints $[9,10]$, whose activation may allow a suppression of lymphocyte functions and of their anticancer efficacy, and the most important of them would be represented by programmed death-1 (PD-1) and its ligands, PD-L1 and PD-L2. Therefore, the block of immune checkpoints by checkpoint inhibitors, such as the anti-PD-1 monoclonal antibodies (MAB), may enhance the efficacy of the anticancer immune response. By synthetizing, the antitumor immunity may be enhanced through two fundamental strategies, consisting of the increase in lymphocyte number and the inhibition of immune checkpoint activities, mainly PD-1 and its ligands. Several cytokines have been employed to enhance the antitumor immune response, including interferon (IFN)alpha, IFN-gamma, IL-2, IL-4, IL-12, and at present the most active antitumor cytokines in humans have appeared to be IL-2 [11] and IL-12 [12]. Moreover, it has to be remarked that the immunotherapy of cancer with IL-2 would represent the only antitumor immunotherapeutic strategy, whose efficacy may be predicted on the basis not only of the biological characteristics of tumors, including histology, tumor genetic features, and disease extensions but also on the basis of 
patient immunobiological response $[13,14]$. In fact, the efficacy of IL-2 immunotherapy in determining tumor regression or at least a chronic stabilization of the neoplastic growth is associated with an increase in lymphocyte count and in eosinophil number $[13,14]$. In more detail, IL-2-induced eosinophilia is generally associated with a stabilization of disease only, while lymphocytosis may predict tumor regression. In any case, tumor eosinophil infiltration may also contribute to tumor cell destruction through the release of cytotoxic proteins, such as the major basic protein (MBP).The main limit of IL-2 cancer immunotherapy has appeared to be represented by the possible concomitant induction of immunosuppressive events, mainly consisting of the activation of the macrophage system [14], which may suppress the antitumor immunity by inducing a chronic inflammatory response [5,7], and T reg cell generation [15], as confirmed by the fact that a low increase in soluble IL-2 receptor as a biomarker of macrophage activation [6), and in $\mathrm{T}$ reg cell count, is associated with an efficacy of treatment $[18,19)$. Therefore, the major problem of IL-2 cancer immunotherapy is represented by the control of the concomitant generation of suppressive events, in particular, T reg cell generation. Several cytokines have been evaluated to amplify IL-2 anticancer efficacy [16-19], but at present the only cytokine, which has appeared to enhance IL-2 anticancer biological response, has been proven to be IL-12, that may increase IL-2 anticancer potency through several mechanisms, including [12]: 1) stimulation of $\mathrm{TH} 1$ differentiation, with a further enhanced endogenous production of IL-2; 2) inhibition of IL-2-induced generation of T reg cells and their TGF-beta production [19); 3) activation of cytotoxic T lymphocytes; 4): anti-angiogenic activity [12]. As far as the other possible anticancer immunotherapeutic strategy, consisting of the block of immune checkpoints, is concerned, in contrast to the clinical behavior followed for the previous IL-2 cancer immunotherapy, the possible prognostic factors predictive of the efficacy of therapy have been researched up to now only within the characteristics of cancer cells and tumor microenvironment, instead of in the immunobiological response of cancer patients, and at present, even though the results are still controversial, the main prognostic marker to predict the efficacy of checkpoint inhibitors, such as the anti-PD-1 MABs, would be represented by the high expression of PD-L1 by tumor cells or tumor microenvironment [20], whereas the treatment with anti-PD-1 inhibitors could be potentially detrimental in the absence of PD-L1 expression. PD-L1 may be expressed by both immune cells and tumor cells, while that of PD-L2would be more specific for the only immune cells, including lymphocytes and macrophages. PD-1 is mainly expressed by both circulating and tumor-infiltrating $T$ lymphocytes, and PD-1 expression is a sign of their immunosuppressive status. On the other hand, PD-L1 and PDL2 are namely expressed by macrophages, particularly by those infiltrating tumor mass, and they reflect their immunosuppressive activity by inhibiting $T$ lymphocyte functions. Since PD-1 expression may influence lymphocyte and macrophage functions, it is probable that it may also influence LMR values. Therefore, a block of PD-1 expression or that of its ligands could improve LMR by stimulating $T$ lymphocyte proliferation and functions and by inhibiting the immunosuppressive action of macrophages and $\mathrm{T}$ reg cells. Unfortunately, no study has been performed up to now to investigate the influence of checkpoint inhibitors on the biological response of cancer patients, including lymphocyte and monocyte number and functionless. Obviously, the possibility to identify patient biological response-related biomarkers predictive of the efficacy of the various anticancer immunotherapies would mainly depend on the knowledge of the physiopathology of the antitumor immunity in humans, as achieved in the last years from both experimental and clinical points of view. The present study was carried out to evaluate the changes occurring in lymphocyte, monocyte and eosinophil numbers under immunotherapy with the antiPD-1 MAB Nivolumab, a fully human lgG4 anti-PD- MAB, by correlating the results with the clinical response and by comparing the results obtained with Nivolumab to those observed under IL-2 cancer immunotherapy.

\section{Materials and Methods}

The study included 30 metastatic non-small cell lung cancer (NSCLC) patients, for whom no chemotherapeutic regimen was available because of progression under previous chemotherapies or poor clinical conditions, which were unable to tolerate a chemotherapeutic approach. Nivolumab was injected intravenously at a dose of $3 \mathrm{mg} / \mathrm{kg} \mathrm{b.w}$. at 15day intervals. The results were compared to those obtained in a historical control group of 20 age- and tumor extensionmatched metastatic NSCLC patients treated by subcutaneous (SC) low-dose IL-2, consisting of 6 MIU/day for 5 days/week in the evening for 4 consecutive weeks, followed by a 21 day rest period. Patients were evaluated before and after 3 months of treatment. The clinical response was assessed according to WHO criteria, by repeating the radiological examinations after 3 months of therapy. On the same way, the immunobiological response of patients was investigated by measuring 
lymphocyte, monocyte, and eosinophil numbers and by detecting LMR before, during and after 3 months of therapy through blood collection at 15-day intervals. Lymphocyte count and LMR values were considered to be within the normal range (95\% confidence limits) when they were greater than $1,500 / \mathrm{mm} 3$ and than 2.1, respectively. Data were reported as mean +/- SE, and results were statistically evaluated by the chisquare test, the Student's t-test, the coefficient of correlation, and the analysis of variance, as appropriate.

\section{Results}

The clinical results according to WHO criteria achieved in Nivolumab and in IL-2 groups are reported in Table 1. As shown, significant differences were seen neither in objective tumor regression rate, consisting of a partial response (PR) nor in the disease control (DC). Table 2 shows the clinical response to Nivolumab therapy in relation to the pretreatment values of lymphocyte count and LMR. Abnormally low values of lymphocyte less than $1,500 / \mathrm{mm} 3$ and of LMR less than 2.1 were seen in 20/30 (67\%) and in 21/30 (70\%) patients, respectively. No significant difference was seen in partial response (PR) percentage between patients with normal or low lymphocyte count prior to therapy. On the same way, no significant difference in PR rate occurred between patients with normal or low pretreatment values of LMR. On the contrary, the percentage of disease control (DC) by taking together patients with tumor regression or stable disease (SD) was higher in patients with normal lymphocyte count and with normal LMR values prior to therapy than in those with abnormal pretreatment values, even though the difference did not reach the statistical significance. Moreover, lymphocyte increase greater than $30 \%$ and monocyte decline greater than $30 \%$ with respect to the pretreatment values occurred on
Nivolumab therapy in $8 / 30$ (27\%) patients and in 6/30 (20\%) patients, respectively, without any significant correlation between lymphocyte increase and monocyte decline $(r=-0.2)$. The clinical response to Nivolumab in relation to lymphocyte, monocyte, and eosinophil count variations on treatment is reported in Table 3. Patients with lymphocyte increase greater than $30 \%$ showed a significantly higher percentage of both $P R(P<0.01)$ and $D C(P<0.05)$ than those, who had no lymphocyte increase. On the same way, the percentages of both PR and DC were significantly higher in patients who showed a decline in monocyte count greater than $30 \%$ on Nivolumab treatment than in those who had no decrease in monocyte count, without, however, statistically significant differences. Finally, Table 4 shows mean values of lymphocyte, monocyte, eosinophil counts and LMR before and after 3-month therapy in patients treated by Nivolumab or IL-2. No significant difference in lymphocyte, monocyte, eosinophil, and LMR mean values were observed before and after therapy in Nivolumab group. However, by considering the variation on therapy in relation to the clinical response, lymphocyte and eosinophil mean numbers increased, whereas monocyte mean count decreased after therapy in the only patients who achieved a PR or an SD, even though none of these differences was statistically significant. On the contrary, LMR mean values and eosinophil mean count significantly increased after therapy in patients who obtained a PR $(P<0.05)$. On the other side, a significant increase in both lymphocyte and eosinophil mean counts occurred in the overall patients treated by IL-2 irrespectively of their clinical response $(p<0.05)$, and the increase was more evident in patients who achieved a PR $(P<0.01)$. Lymphocyte, monocyte, and eosinophil mean values found before and after therapy in patients treated with Nivolumab or IL-2 are illustrated in Figure 1, Figure, Figure 3, and Figure 4.

Table 1: Clinical response (WHO criteria) to Nivolumab and to SC low-dose IL-2 in metastatic non-small cell lung cancer (NSCLC) patients.

\begin{tabular}{|l|c|c|c|c|c|c|c|}
\hline \multirow{2}{*}{ Patients } & \multicolumn{9}{|c|}{ Clinical Response* } \\
\cline { 2 - 8 } & $\mathbf{n}$ & CR & PR & CR+PR & SD & DC(CR+PR+SD) & PD \\
\hline NIVOLUMAB & 30 & 0 & 6 & $6(20 \%)$ & 14 & $20(67 \%)$ & $10(33 \%)$ \\
\hline IL-2 & 20 & 0 & 4 & $4(20 \%)$ & 10 & $14(70 \%)$ & $6(30 \%)$ \\
\hline
\end{tabular}

CR: Complete Response; PR: Partial Response; SD: Stable Disease; DC: Disease Control; PD: Progressive Disease.

Table 2: Clinical response (WHO criteria) in Nivolumab group in relation to the pretreatment values of LMR and lymphocyte count.

\begin{tabular}{|l|c|c|c|c|c|c|c|}
\hline \multirow{2}{*}{ Parameter } & \multicolumn{9}{c|}{ Clinical Response* } \\
\cline { 2 - 8 } & $\mathbf{n}$ & CR & PR & CR+PR & SD & DC(CR+PR+SD) & PD \\
\hline Lymphocyte Count & & & & & & &
\end{tabular}




\begin{tabular}{|c|c|c|c|c|c|c|c|}
\hline less than $1,500 / \mathrm{mm} 3$ & 20 & 0 & 4 & $4(20 \%)$ & 8 & $12(60 \%)$ & $8(40 \%)$ \\
\hline more than $1,500 / \mathrm{mm} 3$ & 10 & 0 & 2 & $2(20 \%)$ & 6 & $8(80 \%)$ & $2(20 \%)$ \\
\hline \multicolumn{8}{|l|}{ LMR } \\
\hline less than 2.1 & 21 & 0 & 4 & 4 (19\%) & 8 & $12(57 \%)$ & $9(43 \%)$ \\
\hline more than 2.1 & 9 & 0 & 2 & $2(22 \%)$ & 6 & $8(89 \%)$ & $1(11 \%)$ \\
\hline
\end{tabular}

CR: Complete Response; PR: Partial Response; SD: Stable Disease; DC: Disease Control; PD: Progressive Disease.

Table 3: Clinical response (WHO criteria) to Nivolumab in relation to lymphocyte, monocyte, eosinophil count and lymphocyte-to-monocyte ratio (LMR) variations on treatment.

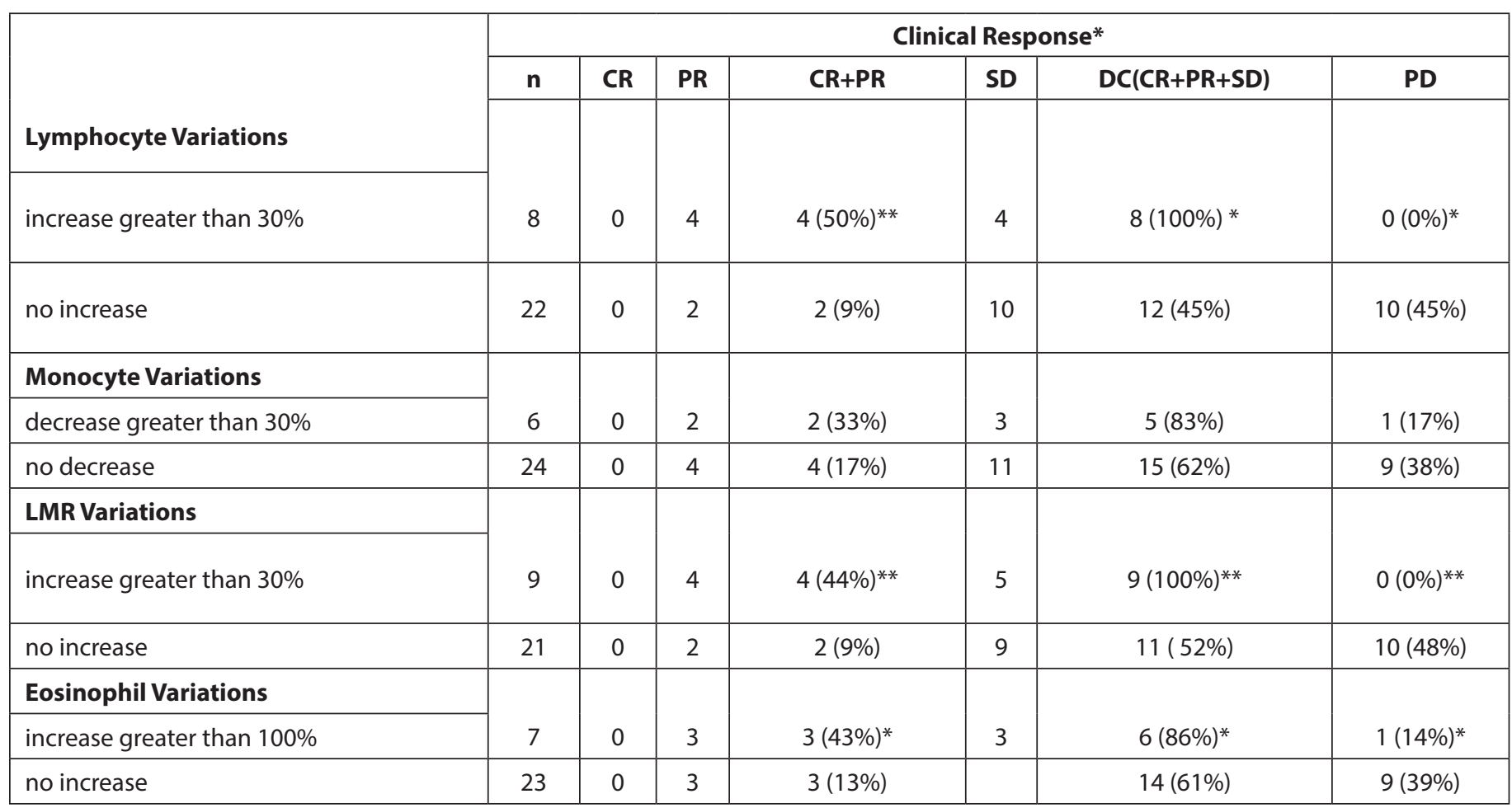

${ }^{* P}<0.05$ vs no increase; ${ }^{* *} \mathrm{P}<0.01$ vs no increase

Table 4: Mean values (+/- SE) of lymphocyte, monocyte, eosinophil counts and of LMR before and after 3 months of therapy with Nivolum$\mathrm{ab}$ or IL-2 in the overall patients and in relation to their clinical response.

\begin{tabular}{|c|c|c|c|c|c|c|c|c|c|}
\hline \multirow{2}{*}{ Therapy } & \multirow{2}{*}{$\mathbf{n}$} & \multicolumn{2}{|c|}{ Lymphocytes } & \multicolumn{2}{|c|}{ Monocytes } & \multicolumn{2}{|c|}{ Eosinophils } & \multicolumn{2}{|c|}{ LMR } \\
\hline & & Before & After & Before & After & Before & After & Before & After \\
\hline \multicolumn{10}{|l|}{ NIVOLUMAB } \\
\hline \multirow[t]{2}{*}{ All Patients } & $30 x$ & 1449 & 1474 & 851 & 834 & 141 & 197 & 1.8 & 1.9 \\
\hline & $+/-\mathrm{SE}$ & 98 & 104 & 63 & 58 & 17 & 24 & 0.1 & 0.2 \\
\hline \multirow[t]{2}{*}{ PR } & $x$ & 1487 & 1810 & 813 & 658 & 153 & $292^{*}$ & 1.8 & $2.6^{*}$ \\
\hline & $+/-S E$ & 223 & 219 & 105 & 22 & 29 & 35 & 0.1 & 0.2 \\
\hline \multirow[t]{2}{*}{ SD } & $\mathrm{X}$ & 1466 & 1676 & 803 & 711 & 143 & 201 & 1.9 & 2.1 \\
\hline & $+/-\mathrm{SE}$ & 109 & 115 & 88 & 93 & 28 & 37 & 0.3 & 0.2 \\
\hline \multirow[t]{2}{*}{ PD } & $x$ & 1398 & 1103 & 902 & 1115 & 134 & 128 & 1.6 & 1.1 \\
\hline & $+/-\mathrm{SE}$ & 115 & 126 & 88 & 121 & 26 & 34 & 0.2 & 0.3 \\
\hline \multicolumn{10}{|c|}{ IL-2 THERAPY } \\
\hline All Patients & $20 x$ & 1531 & $2078^{*}$ & 805 & 756 & 152 & $276^{*}$ & 1.7 & 1.9 \\
\hline
\end{tabular}

Citation: Lissoni P (2019). A Clinical Study of Biomarkers to Predict the Efficacy of Cancer Immunotherapy with Subcutane-

ous Low-Dose II-2 or with Immune Checkpoint Inhibitors. Oncogen 2(2): 9. 


\begin{tabular}{|l|c|c|c|c|c|c|c|c|c|}
\hline & $+/-S E$ & 95 & 128 & 66 & 82 & 26 & 31 & 0.2 & 0.2 \\
\hline \multirow{3}{*}{ PR } & $\mathrm{X}$ & 1675 & $2615^{* *}$ & 786 & 697 & 163 & $338^{* *}$ & 1.8 & $2.5^{*}$ \\
\cline { 2 - 11 } & $+/-S E$ & 134 & 208 & 91 & 104 & 35 & 54 & 0.1 & 0.2 \\
\hline \multirow{3}{*}{ SD } & $\mathrm{X}$ & 1558 & 2121 & 801 & 738 & 158 & $356^{* *}$ & 1.7 & 1.9 \\
\cline { 2 - 11 } & $+/-S E$ & 108 & 245 & 88 & 95 & 33 & 51 & 0.2 & 0.1 \\
\hline \multirow{2}{*}{ PD } & $\mathrm{X}$ & 1409 & 1514 & 867 & 908 & 144 & 166 & 1.6 & 1.3 \\
\cline { 2 - 11 } & $+/-S E$ & 117 & 165 & 76 & 101 & 31 & 54 & 0.2 & 0.2 \\
\hline
\end{tabular}

${ }^{*} \mathrm{P}<0.05$ vs before; ${ }^{*} \mathrm{P}<0.01$ vs before

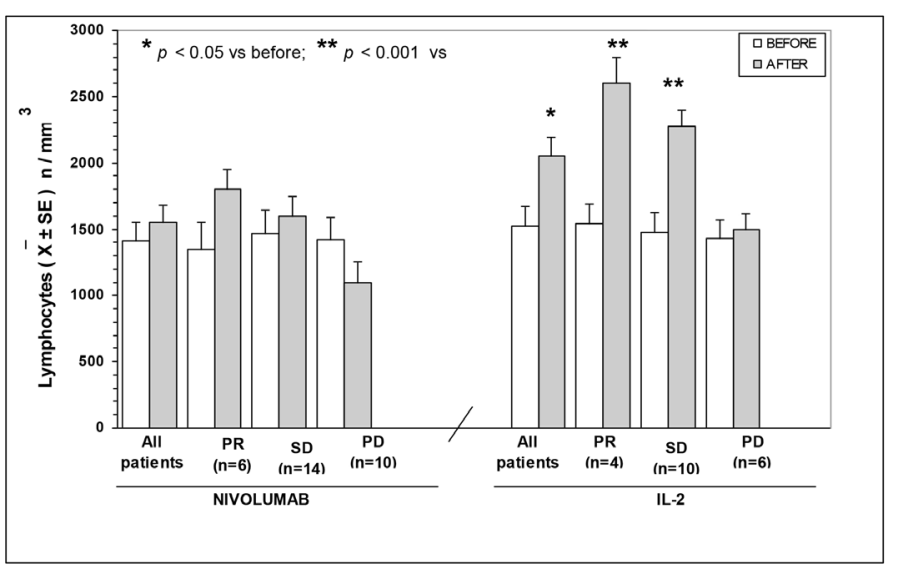

Figure 1: Lymphocytes mean number before and after Nivolumab or IL2

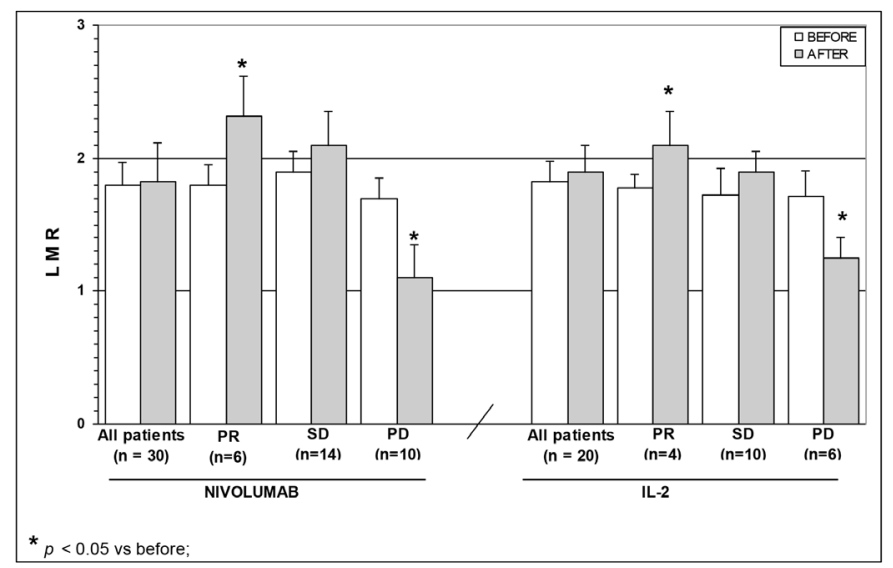

Figure 3: Lymphocytes to monocytes ratio (LMR) mean number before and after Nivolumab or IL2

\section{Discussion}

In addition to the potential importance of tumor and tumor microenvironment expression of biomarkers, such as PD-L1 [20], this preliminary study would suggest that the efficacy of cancer immunotherapies with immune checkpoint inhibitors, namely the anti-PD-1 MABs, or with antitumor cytokines, such as IL-2, may be predicted also on basis of patient immunobiological response by monitoring the changes in the main immune cells involved in regulating the antitumor immunity, which is stimulated by lymphocytes and suppressed by the macrophage system through a possible direct stimulatory effect on cancer cell proliferation and a promoting action of $\mathrm{T}$ reg lymphocyte generation [1-5], as

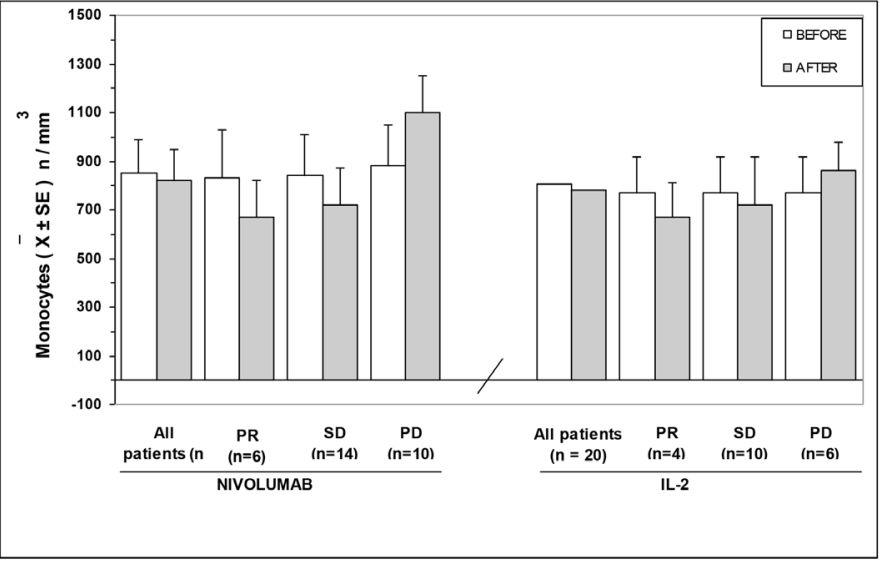

Figure 2: Monocytes mean number before and after Nivolumab or IL2

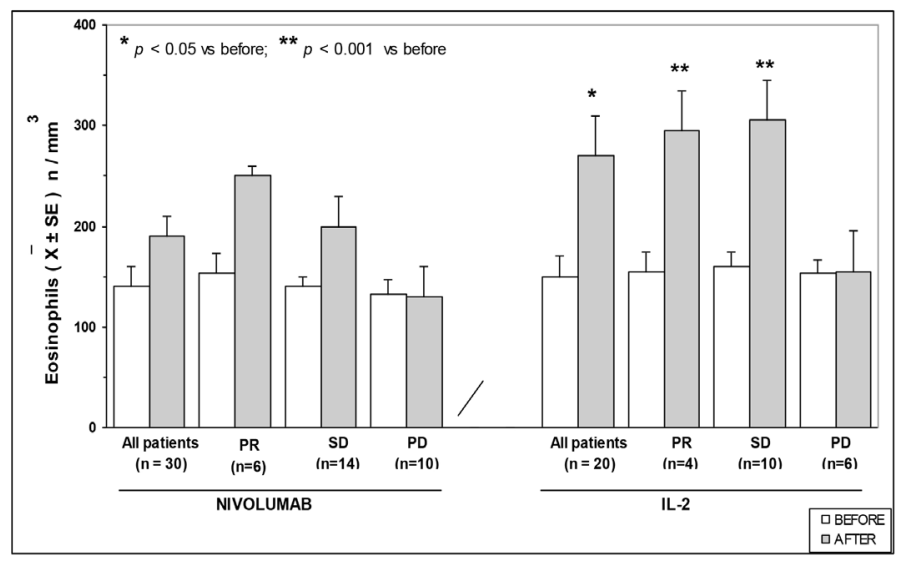

Figure 4: Eosinophils mean count before and after Nivolumab or IL2

reflected by the simple LMR values [8]. According to the results of this preliminary study the possible biomarkers predictive of the efficacy of the various anticancer immunotherapies are the same for both IL-2 immunotherapy and immunotherapy with an anti-PD-1 MAB, such as Nivolumab, and that they would mainly consist of an increase in lymphocyte and eosinophil numbers and a decrease in monocyte count, with the following increase in LMR values. The main difference in the immunobiological effects induced by IL-2 and Nivolumab is consisting of the more evident increase in lymphocyte and eosinophil count in most patients under IL-2 therapy, whereas it substantially occurs in the only patients with tumor regression or at least a disease stabilization during Nivolumab 
immunotherapy. Moreover, this study would suggest that the evidence of a normal lymphocyte count prior to therapy, and more specifically that of a normal pretreatment value of LMR, would predict the efficacy of the immunotherapy, at least in terms of disease stabilization. Therefore, the results of this study seem to suggest that cancer immunotherapy, irrespectively of the type of strategy, had to profoundly modify the endogenous immune status to be effective in blocking tumor growth, by stimulating the proliferation and activation of antitumor lymphocytes and by concomitantly suppressing the activity of the macrophage system and the consequent chronic inflammatory response-related $\mathrm{T}$ reg cell generation $[5,7]$. By separately considering the immunotherapies of cancer with II-2 or with PD-1 inhibitors, it has to be remarked that the single strategy would mainly act on only one of the two main fundamental variables responsible for the efficacy of the antitumor immunity, consisting of the activation of lymphocyte functions and the suppression of macrophage activity. In more detail, IL-2 immunotherapy would mainly act by inducing proliferation and activation of $\mathrm{T}$ lymphocyte system, whereas the anti-PD-1 strategies would be more effective in blocking macrophage-related suppression of the antitumor immunity, while their impact on lymphocyte count and functionless is less relevant. Therefore, the association between IL-2 immunotherapy to enhance lymphocyte count and activation and cancer immunotherapy with antiPD-1 molecules to inhibit the macrophage system and PD-1 expression by $\mathrm{T}$ reg lymphocytes could allow most effective therapeutic results with respect to the single agents, as confirmed by preliminary experimental results [21]. IL2-induced generation of $\mathrm{T}$ reg lymphocytes may be also counteracted by IL-12 [19]. On the same way, PD-1 expression, as well as that of other immune checkpoints, could be also under a neuroimmune regulation, and in particular it has been shown that the evolution of monocytes into macrophages provided by immunosuppressive activity is associated with a lack of expression of monocyte receptor for the pineal hormone melatonin, whose antitumor immunostimulatory activity has been well demonstrated [22]. In conclusion, this study would suggest that the efficacy of both cancer immunotherapies with IL-2 or with PD-1 inhibitors may depend and be predicted not only on the basis of tumor cell and tumor microenvironment-related biological characteristics but also on the basis of the endogenous immune status of patients and its variations on treatment, and in particular it would depend on the balance between lymphocyte and macrophage systems, as synthetized by LMR. Therefore, from a clinical point of view, it seems to be sufficient to monitor LMR values prior to therapy and on treatment, and in particular, the evidence of a normal pretreatment value of LMR or a progressive normalization on therapy is associated with a high probability to obtain at least a disease stabilization. The advantage to predict the efficacy of cancer immunotherapies on the basis of patient biological immuno-inflammatory response itself instead of the only tumor-related biomarkers is consisting of the possibility to pilot the biological response in an antitumor way in the presence of a low lymphocyte response and/or an exaggerated macrophage response by associating other potential immunomodulating agents, such as IL-2 itself in patients with lymphocytopenia under antiPD-1 therapies, as well as the same PD-1-inhibitors in patients presenting an excessive $\mathrm{T}$ reg- and macrophage-mediated immunosuppressive reaction under therapy with IL-2. In particular, the apparent lower efficacy of PD-1 inhibitors in the presence of lymphocytopenia prior to therapy could be corrected by a previous short-time injection of SC low-dose IL-2, as observed in experimental conditions [21].Cancer chemotherapy itself may act not only through a cytotoxic mechanism, but also by modulating the antitumor immunity and the cytokine network, and in particular it has been shown that low-dose adriamycin may stimulate IL-2 production, cyclophosphamide may counteract $T$ suppressor lymphocyte generation, gemcitabine may stimulate IL-12 production by modulating macrophage differentiation, and cisplatin may decrease IL-6 levels [23]. Therefore, irrespectively of the type of treatment, the efficacy of each anticancer therapy, including immunotherapy and chemotherapy itself, would be associated with an improvement in antitumor immune function, with an increase in lymphocyte activity and a decrease in the monocyte-macrophage one, as reflected by the LMR [8]. Therefore, LMR could constitute a simple biomarker reflecting the immune status of patients and provided by a prognostic significance either in basal conditions or during the different anticancer therapies. In any case, further studies by monitoring cytokines levels and lymphocyte subsets will be required to better define the immunobiological mechanisms involved in determining the efficacy of the various anticancer therapies and in particular the antitumor immunotherapies.

\section{References}

1. Riesco A (1970) Five-year cancer cure: relation to total amount of lymphocytes and neutrophils. Cancer 25(1):135-140

2. Grimm EA, Mazumder A, Zhang HZ, Rosenberg SA (1982) Lymphokine-activated killer cell phenomenon. Lysis of natural killer-resistant fresh solid tumor cells by 
interleukin 2-activated autologous human peripheral blood lymphocytes. J Exp Med 155(6):1823-1841

3. Zou W (2006) Regulatory T cells, tumor immunity and immunotherapy. Nat Rev Immunol 6(4):295-307

4. Kim R, Emi M, Tanabe K, Uchida Y, Toge T (2004) The role of Fas ligand and transforming growth factor beta in tumor progression: molecular mechanisms of immune privilege via Fas-mediated apoptosis and potential targets for cancer therapy. Cancer 100)11):2281-2291

5. Mantovani A, Allavena P, Sica A, Balkwill F (2008) Cancerrelated inflammation. Nature 454(7203):436-444

6. Lissoni $P$ (1996) Prognostic markers in interleukin-2 therapy. Cancer Biother Radiopharm 11[5):285-287

7. Grivennikov SI, Greten FR, Karin M (2010) Immunity, inflammation, and cancer. Cell 140[6):883-899

8. Eo WK, Chang HJ, Kwon SH, Koh SB, Kim YO, Ji Yl, et al. (2016) The lymphocyte-to-monocyte ratio predicts patient survival and aggressiveness of ovarian cancer. J Cancer 7[3):289-296

9. Ashwell S, Janetka JW, Zabludoff S (2008) Keeping checkpoint kinases in line: new selective inhibitors in clinical trials. Expert Opin Investig Drugs 17(9):1331-1340

10. Chen J, Jiang CC, Jin L, Zang XD [2016) Regulation of PDL1: a novel role of pro-survival signalling in cancer. Ann Oncol 27(3):409-416

11. Whittington R, Faulds D [1993) Interleukin-2. A review of its pharmacological properties and therapeutic use in patients with cancer. Drugs 46(3):446-483

12. Banks RE, Patel PM, Selby PJ (1995) Interleukin-12: a new clinical player in cytokine therapy. Br J Cancer 71(4):655659

13. Atzpodien J, Kirchner H (1990) Cancer, cytokines and cytotoxic cells: interleukin-2 in the immunotherapy of human neoplasms. Klin Wochenschr 68(1):1-11

14. Fumaglli L, Lissoni P, Di Felice G, Meregalli S, Valsuani G, Mengo S, Rovelli F (1999) Pretreatment serum markers and lymphocyte response to interleukin-2 therapy. $\mathrm{Br} J$
Cancer 80(3):407-411

15. Cesana GC, DeRaffaele G, Cohen S, Moroziewicz D, Mitcham J, Stoutenburg J, et al. [2006) Characretization of $\mathrm{CD} 4+\mathrm{CD} 25$ + regulatory $T$ cells in patients treated with high-dose interleukin-2 for metastatic melanoma or renal cell carcinoma. J Clin Oncol 24(7):1169-1177

16. Foon KA (1989) Biological response modifiers: the new immunotherapy. Cancer Res 49(7):125-127

17. Atkins MB, Vachino G, Tilg HJ, Karp DD, Robert NJ, Kappler K, et al. (1992) Phase I evaluation of thrice-daily intravenous bolus interleukin-4 in patients with refractory malignancy. J Clin Oncol 10(11):1802-1809

18. Waldmann TA (2014) Interleukin-15 in the treatment of cancer. Expert Rev Clin Immunol 10(12):1689-1701

19. Prochazkiva J, Pokoma K, Holan V (2012) IL-12 inhibits the TGF-beta-dependent $\mathrm{T}$ cell developmental programs and skews the TGF-beta-induced differentiation into a Th1-like direction. Immunobiology 217(1):74-82

20. Iway $Y$, Ishida $M$, Tanaka $Y$, Okazaki T, Honjo T, Minato N (2002) Involvement of PD-L1 on tumor cells in the escape from host immune system and tumor immunotherapy by PD-L1 blockade. Proc Natl Acad Sci USA 99(19): 1229312297

21. West EE, Jin HT, Rasheed AU, Penaloza-MacMaster P, Ha SJ, Tan WG, et al. (2013) PD-L1 blockade synregize with IL-2 therapy in reinv igorating exhausted T cells. J Clin Invest 123(6):2604-2615

22. Lissoni $P$ (1999) The pineal gland as a central regulator of cytokine network. Neuro Endocrinol Lett 20(6):343-349

23. Ehrke MJ, Mihich E, Berd D, Mastrangelo MJ (1989) Effects of anticancer drugs on the immune system in humans. Semin Oncol 16(3):230-239

Copyright: Lissoni P, et al. @2019. This is an open-access article distributed under the terms of the Creative Commons Attribution License, which permits unrestricted use, distribution, and reproduction in any medium, provided the original author and source are credited. 